\section{$\S 19$. Control of 3D Edge Radiation Structure with Resonant Magnetic Perturbation Fields Applied to Stochastic Layer and Stabilization of Radiative Divertor Plasma in LHD}

Kobayashi, M., Masuzaki, S., Yamada, I., Tamura, N., Akiyama, T., Narushima, Y., LHD Experiment Group, Ohno, N. (Nagoya Univ.)

Divertor power load reduction is one crucial issue for magnetically confined fusion reactors. Increased edge radiation can dissipate power before reaching divertor plates. Stable sustainment of such enhanced radiation, i.e. radiative divertor (RD) or detached divertor, is, however, still an open issue. In this paper, we present experimental evidence of edge radiation stabilization effect and potential of divertor power load control with resonant magnetic perturbation (RMP).

Figure 1 shows time evolution of peak divertor power load, radiation intensity $\left(P_{r a d}\right)$ and line averaged density $\left(\bar{n}_{e}\right)$ during density ramp up experiments with and without RMP. RMP has $m / n=1 / 1$ mode, which has resonance layer in the edge stochastic region, and creates remnant island. The perturbation strength is kept constant at $\widetilde{b}_{r} / B_{0} \approx 0.1 \%$ throughout the discharge in the case with RMP. The plasma is heated by neutral beam injection (NBI) with $\sim 8 \mathrm{MW}$ of deposited power in both cases. The divertor power load is estimated with Langmuir probe. The radiation is obtained with photo diode array viewing almost entire plasma at specific toroidal location. Without RMP (gray lines), the radiation intensity gradually increases with increasing density. The rapid increase of radiation intensity at $t \sim 3.8 \mathrm{sec}$ with concomitant density rise indicates onset of thermal instability. The instability grows so rapidly that it is difficult to stabilize the density rise, leading to discharge termination. With RMP (black lines), on the other hand, transition to enhanced radiation state occurs at $t \sim 3 \mathrm{sec}$, and it leads to divertor power load reduction by a factor of $3 \sim 10$. The RD operation is successfully sustained by gas puff feedback control up to the end of NBI. The results show stabilization effect of RMP on the radiating edge plasma. The enhanced radiation with RMP is due to increased volume of low $T_{e}$ $(\sim 10 \mathrm{eV})$ region caused by temperature flattening at the Opoint. 3D edge transport simulation result, which is consistent with the radiation profile measurement, show that the radiation increases further around X-point of the island (1), where the code predicts $n_{e}>10^{20} \mathrm{~m}^{-3}$ and $T_{e} \sim \mathrm{a}$ few eV. The well structured edge radiation with RMP such as the selective cooling around X-point is considered to provide stabilization effect by holding the intense radiation there and thus avoids it penetrating inward.

Fig.2 shows dependence of controllability of RMP assisted RD on radial location of the island X-point,
$r_{X \text { point }}-r_{L C F S}$, and on RMP strength, $\widetilde{b}_{r} / B_{0}$, where $r_{X \text { point }}$ and $r_{L C F S}$ are minor radii of the X-point and last closed flux surface. The controllable and uncontrollable cases mean the successful RMP assisted RD and radiative collapse even with RMP, respectively. It is found that stable $\mathrm{RD}$ is realized when $r_{X \text { point }}-r_{\text {LCFS }} \geq 0.05 \mathrm{~m}$. The radial extent of intense radiation at X-point is estimated at several $\mathrm{cm}$ in the $3 \mathrm{D}$ simulation. The results suggest importance of separation of radiation layer from confinement region for stable RD. Threshold of $\widetilde{b}_{r} / B_{0}$ is found to be $5 \times 10^{-4}$, corresponding to island width $w_{\text {vac }} / a \approx 0.23\left(w_{\text {vac }} \approx 0.19 \mathrm{~m}\right)$ at outboard midplane. In this low RMP strength, despite the still substantial size of $w_{v a c}$, almost no $T_{e}$ flattening is observed, indicating plasma healing of island. Thus radiation enhancement with the island structure is not available, resulting in radiative collapse.

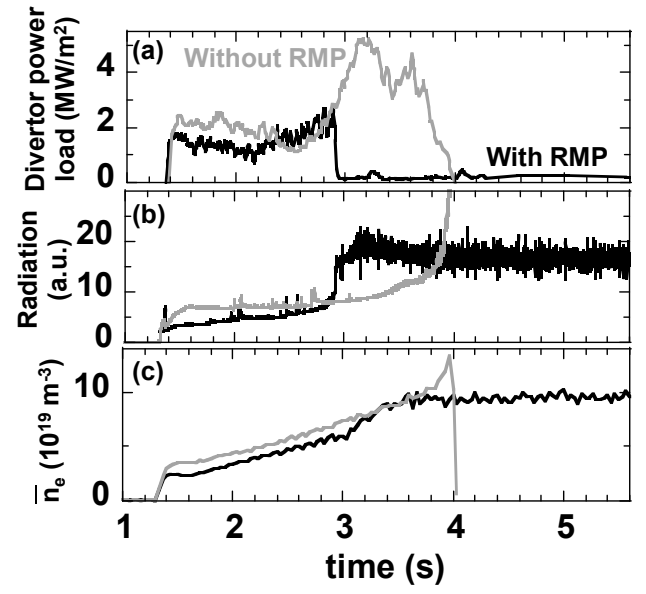

Fig.1 Time evolution of (a) peak divertor power load, (b) $P_{\text {rad }}$ and (c) $\bar{n}_{e}$. Black lines: with RMP, gray lines: without RMP.

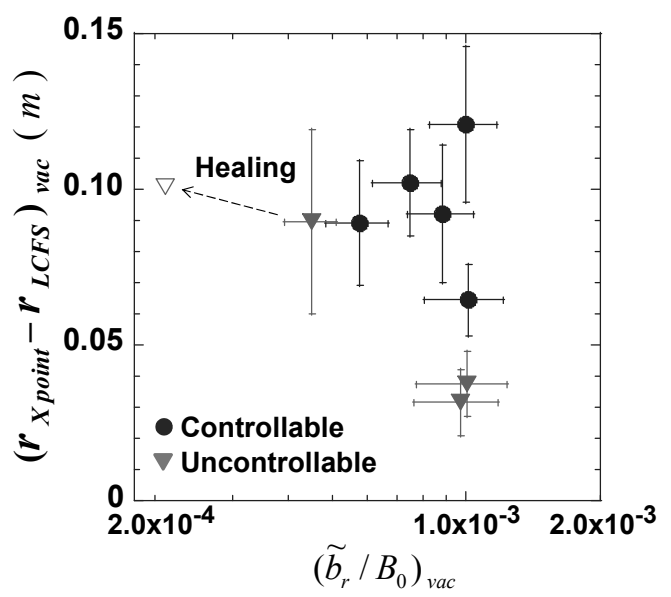

Fig.2 Dependence of RMP assisted RD controllability on magnetic field configuration. $\boldsymbol{\nabla}$ : uncontrollable, $\mathbf{O}$ : controllable.

1) Kobayashi, M., Masuzaki, S. et al., Physics of Plasmas 17 (2010) 056111. 